\title{
Die Digitalisierung des Verkehrs - Mobilität 4.0
}

\author{
H. Malleck OVE, C. Mecklenbräuker
}

Online publiziert am 16. Oktober 2015

(c) Springer Verlag Wien 2015

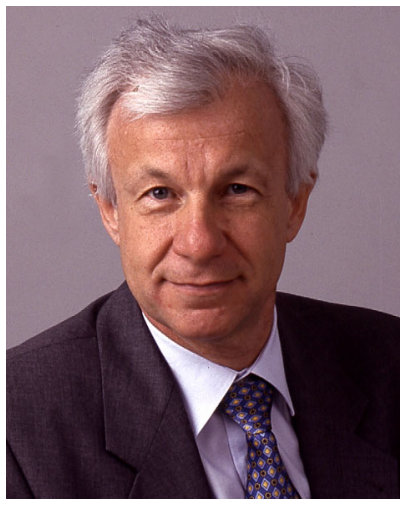

Dipl.-Ing. Dr. Helmut Malleck

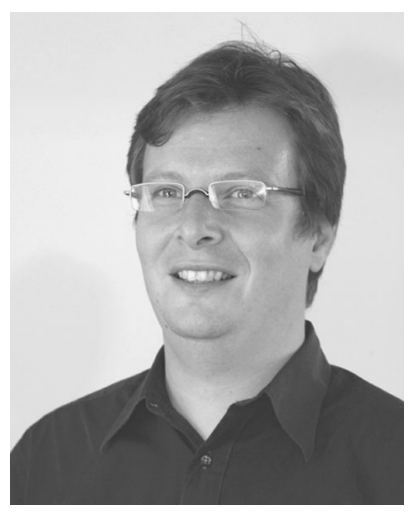

Univ.-Prof. Dipl.-Ing. Dr.-Ing. Christoph Mecklenbräuker

\begin{abstract}
Liebe Leserin und lieber Leser,

eine entsprechende Verkehrsanbindung zählt heutzutage neben sicherer Energieversorgung und der Möglichkeit, große Datenmengen zu übertragen, zu den wesentlichen Standortfaktoren industrieller Wertschöpfung. Dazu kommt, dass regionale, nationale, aber auch globale Mobilitätsbedürfnisse - sowohl im Personen- als auch im Güterverkehr - in einem vor Jahrzehnten ungeahnten Ausmaß angestiegen sind. Dies alles, überlagert von stetig fortschreitender Urbanisierung und vielerorts beeinflusst durch eine alternde Gesellschaft, macht die Modernisierung und Weiterentwicklung im StraßenSchienen-, Wasser- und Luftverkehr unumgänglich notwendig. Dafür sind innovative Gesamtverkehrskonzepte, bei denen öffentlicher und privater Personenverkehr vernetzt werden, Voraussetzung für effiziente und sichere Mobilität.
\end{abstract}

„Mobilität 4.0" zeigt Lösungswege im Wechselspiel zwischen limitierten Transportkapazitäten und steigenden Anforderungen an Mobilitätsservices auf, um einen Ausgleich zwischen Transportnutzen und Transportnotwendigkeit herbeizuführen. Einer bedarfsgerecht harmonisierten Verkehrssteuerung auf Basis aktueller Verkehrsdaten kommt dabei, neben der Implementierung innovativer intelligenter Transportsysteme, überragende Bedeutung zu. Die Fragestellungen zur Implementierung innovativer intelligenter Fahrassistenzsysteme, etwa mit laserund lichtgesteuerter Umfelderkennung, bleiben im vorliegenden Themenheft weitgehend ausgespart, ebenso wie pilotiertes Fahren und Parken, weiterer Ausbau der Elektromobilität und beispielsweise der vernetzte Einsatz hoch-verfügbarer und ausfallssicherer Systeme im Schienen- und Luftverkehr.

Die Digitalisierung des Verkehrs als eine Facette von Mobilität 4.0 soll im vorliegenden Themenheft näher beleuchtet werden. Dazu wurde bereits beim heurigen „Informationstechnischen Kolloqui- um" (Wien, 19. Mai 2015) berichtet. Beispielsweise wurde ein Modell für den Straßenverkehr in Tschechien und in der Slowakei vorgestellt, welches Verkehrsflüsse und -staus sowie Verzögerungen im Straßennetz als Quelldaten erfasst. Durch zeitliche und räumliche Big Data-Auswertungen kann man Verkehrsstörungen proaktiv begegnen und den Mobilitätsbedarf tages- und uhrzeitabhängig, aber auch abhängig von äußeren Umständen, wie Wetterlage oder Großveranstaltungen, ermitteln.

Wer im Verkehrsgetümmel beispielsweise von São Paulo, Brasilien, unterwegs ist, weiß, wie unverzichtbar vor Fahrtantritt die Stauinfos der Navi-Apps sind. Aber, ob dort jemals durch Echtzeitauswertung von Verkehrsdaten eine Beeinflussung des Straßenverkehrs möglich wird, darf bezweifelt werden. In knappstem Abstand folgt Auto auf Auto, und im Verkehrsgeschehen sind Motorräder praktisch überall. Fußgänger queren zumeist nur in Gruppen die Kreuzungsbereiche, eben dann, wenn es der Straßenverkehr gerade zulässt. Zugegeben, auch bei uns läuft im Straßenverkehr nicht alles regelkonform, vor allem sind aber die Prioritäten anders verteilt. Fußgänger haben gegenüber den motorisierten Verkehrsteilnehmern berechtigten Schutz, jedenfalls auf den Zebrastreifen. Lediglich manche Radfahrer sorgen für unliebsame Abwechslung im Verkehrsgeschehen.

Das vorliegende Themenheft der e\&i umfasst acht Originalarbeiten. Zuerst wird auf den technologiebasierten Paradigmenwechsel mit Mobility 4.0 eingegangen, danach werden grundsätzliche Fakten zur Verkehrsentwicklung herausgearbeitet. Die weiteren Beiträge widmen sich recht unterschiedlichen Aspekten: der Modernisierung und Leistungssteigerung im europäischen Schienenverkehr, praktikablen Ansätzen zur Verkehrsbeeinflussung mit digitalen Methoden, der Errichtung eines kooperativen intelligenten Verkehrssystems quer durch Europa, den sich ergebenden zukünftigen Verkehrsangeboten aus einer digitalen Abbildung des Zusammenwirkens realer Verkehrsmodi sowie den Kommunikationserfordernissen und -standards beim automatisierten Fahren. Mit einer kritischen Betrachtung herkömmlicher Verkehrsflussanalysemethoden schließt der Originalarbeiten-Teil dieser e\&i-Ausgabe ab.

\section{Originalarbeiten zum Themenschwerpunkt}

Reinhard Pfliegl, Hartmut Keller: "Mobility Governance - Digitalisierung des Verkehrs im Kontext zu Industrie 4.0 und der Verantwortung der Gesellschaft zur Nachhaltigkeit der Mobilität"

Das Grundsatzpapier stellt die Entwicklungen der Verkehrssysteme zu künftiger Mobilität 4.0 dar, in Analogie zu den Stufen der Industrialisierung hin zu Industrie 4.0. Industrie 4.0 ermöglicht in der nächsten Dekade auf Basis Cyber-Physischer Systeme und mit dem Internet der Dinge neue Industrieprozesse und Geschäftsmodelle.

Malleck, Helmut, Arbeitskreis für technische Koordination für öffentliche Kommunikationsnetze und -dienste (AK-TK), Postfach 147, 1006 Wien, Österreich (E-Mail: helmut.malleck@oefeg.at); Mecklenbräuker, Christoph, Institute of Telecommunications, Technische Universität Wien, Gußhausstraße 25, 1040 Wien, Österreich 
Die Entwicklung von Industrie 4.0 steht in Wechselwirkung mit den Cyber-Physischen-Verkehrssystemen, mit Mobilität 4.0. Neben dem Handlungsbedarf zur Gestaltung und Finanzierung künftiger Verkehrssysteme mit entsprechender Mobilitätsqualität sind die technische Organisation der Verkehrssysteme und die institutionelle Ebene der Verwaltungsstrukturen gefordert. Dabei ergeben sich Chancen für eine Mobilitätskultur mit nachhaltig dynamischer Balance von Mobilitätsnachfrage und verfügbarer Kapazität. Im Zusammenwirken neuer Strukturen kann sich auch Mobilität als Service unter dem Schirm einer Mobility Governance (Politik, Service Arbieter, Nutzer) etablieren.

Barbara Lenz: "Vernetzung - Revolution für die urbane Mobilität der Zukunft?"

Eingangs wird der aktuell in Städten bestehende dringende Bedarf zur Lösung von Verkehrsproblemen und zum Umgang mit sich wandelnden Mobilitätsbedürfnissen analysiert. Dabei sind Informationsund Kommunikationstechnologien zentrale Technologien zur Umsetzung sowohl des Effizienz- als auch des Nachhaltigkeitszieles, insbesondere im urbanen Verkehr. Die Situation erscheint jedoch ausschließlich mit Technologieeinsatz nicht lösbar. Daher werden Nutzungsbereitschaft und tatsächliche Nutzung der aus der Digitalisierung erwachsenden Möglichkeiten durch die Verkehrsteilnehmer selbst mit in Betracht gezogen. Zu beachten ist dabei, dass sich aktuell der Mobilitätsbedarf und das Verkehrsverhalten in wesentlichen Gruppen der Bevölkerung verändern.

Josef Doppelbauer: "Regulierungs- und Standardisierungsaspekte im europäischen Eisenbahnwesen"

Trotz vieler Vorteile ist der Verkehrsträger Eisenbahn wegen seiner Fragmentierung durch nationale Regeln, nationale Zulassungsverfahren sowie nationale Eisenbahnfahrzeuge und Signaltechnik eingeschränkt. Die Hauptaufgaben der Europäischen Eisenbahnagentur (ERA) liegen auf der Sicherheit des Eisenbahnbetriebs, der Schaffung der Grundlagen für eine europaweite Interoperabilität sowie auf der Systemverantwortung für das europäische Zugsteuerungssystem ERTMS. Die zunehmende Digitalisierung von Prozessen und Produkten im Eisenbahnverkehr bringt in den genannten Bereichen weitere Herausforderungen mit sich. Die Europäische Eisenbahnagentur will zukünftig dazu beitragen, dass der einheitliche europäische Eisenbahnraum gerade im Zeitalter der Digitalisierung Wirklichkeit werden kann.

Birgit Kohla, Martin Fellendorf: "Lenken und Leiten des städtischen Verkehrs"

Mit zunehmender Urbanisierung gerät die städtische Mobilität aufgrund fehlender Flächen und steigender Umweltbelastungen an ihre Grenzen. Die Allokation der Mobilität als knappes Gut ist bisher eine öffentliche Aufgabe, die immer mehr durch private Dienstleistungen ergänzt wird. Hier werden sowohl bekannte als auch innovative Ansätze einer zukunftsweisenden, umweltgerechten Mobilitätsnutzung aufgezeigt, wie beispielsweise die Bevorzugung des Öffentlichen Verkehrs, Zufahrtsbeschränkungen durch Straßenbenützungsgebühren, Parkraummanagement, Carsharing-Angebote, intelligente "Ampeln" und kooperative Fahrzeuge.

Marko Jandrisits et al.: "From research and living lab to implementation of C-ITS"

Der Beitrag beschreibt den Stand der Arbeiten zur Einführung von Kooperativen Intelligenten Verkehrssystemen, Cooperative ITS Corridor (C-ITS), auf Autobahnen im Korridor Rotterdam - Frankfurt - Wien mit Baustellenwarninformationen und Fahrzeugdatenerfassung als erste Anwendungen. Zunächst werden Motivation und
Strategie bei der Umsetzung dieses Vorhabens erläutert. Das Living Lab im Umfeld kooperativer Fahrzeuge stellt dabei einen wichtigen und notwendigen Ansatz dar. Anschließend werden Systemarchitektur und Typen der Roadside ITS Station, Harmonisierungsgründe und generelle Anforderungen sowie die Nachrichtenformate bei C-ITS vorgestellt. Abschließend wird auf Data Exchange Vehicle-toInfrastructure eingegangen.

Martin Russ, Karin Tausz: „Mobilität als Service - Nutzerorientierung als Paradigma zwischen Markt und öffentlicher Grundvorsorge" Der Zugang zu Mobilitätsdaten für neue nutzerorientierte Mobilitätsservices steht im Zentrum der Digitalisierung des Verkehrsystems. Kommt hier der Markt allein zu vernetzten, leistbaren und umweltfreundlichen Mobilitätsangeboten? Welche Aufgaben kommen der Öffentlichen Hand zu? Der Aufbau digitaler Basisinfrastrukturen, das Synchronisieren der digitalen Services mit realen Infrastrukturen setzt öffentlich-private Partnerschaften voraus.

Andreas Festag: "Standards for vehicular communication - from IEEE $802.11 p$ to $5 G^{\prime \prime}$

Die Standardisierung für drahtlose Fahrzeugkommunikationen muss Interoperabilität sicherstellen, Regulatorien und Gesetzen genügen und mithelfen, weitere Märkte zu erschließen. Anfangs wurden konsistente Standardisierungsreihen zur Vehicle-to-Vehicle- und Vehicleto-Infrastructure-Kommunikation für die Hauptanwendungen Fahrerinformation und Warnungen erstellt, beruhend auf WiFi Standard IEEE 802.11. Sie wurden mit C-ITS in Europa und DSRC in USA bezeichnet. Aus dem Blickwinkel der Standardisierung gibt der Artikel einen Überblick über die Schlüsselprotokolle von C-ITS und DSRC. Weiters werden automatisiertes Fahren - als potenzieller neuer Anwendungsbereich für Fahrzeugkommunikation - analysiert, die Kommunikationserfordernisse diskutiert und mögliche Richtungen für zukünftige Versionen der Fahrzeugkommunikationsstandards hergeleitet.

Boris S. Kerner: "Failure of classical traffic flow theories: a critical review"

Die grundlegende Aussage der Arbeit ist, dass allgemein akzeptierte Theorien und Modelle für Verkehr und Transport mit den empirischen Grundlagen der Verkehrswissenschaft nicht übereinstimmen. Es wird hergeleitet, dass als theoretische Basis für die Entwicklung von zuverlässigem automatisierten Fahren sowie zur Optimierung von Fahrzeugverkehr und Transportnetz, eine unbegrenzte Straßenkapazität zu jedem Zeitpunkt mit einem Satz fundamentaler empirischer Eigenschaften eines Verkehrszusammenbruchs an einer StraBenenge steht.

\section{Themenbeiträge in der Rubrik „Praxis und Wissen“}

Die folgenden fünf Beiträge in der Rubrik „Praxis und Wissen“ machen noch einmal deutlich, welche zum Teil recht weit auseinander liegenden Themenbereiche zur Digitalisierung des Verkehrs gehören. Leider kommt dabei die Abhandlung der rechtlichen Rahmenbedingungen für Mobility 4.0 zu kurz. Das wurde von uns keineswegs vergessen, ist aber in Terminkollisionen schreibwilliger Fachleute und im Timeout für die Hefterstellung begründet. Wir entschuldigen uns dafür und werden das in anderem Zusammenhang nachholen.

Der Österreichische Städtebund berichtet im Beitrag "Smart Cities - neue Strategien als Erfolgsgarant für intelligente und zukunftsorientierte Stadt-Umland-Entwicklung", dass bereits 2014 ca. 50 \% der österreichischen Bevölkerung in Städten wohnten, ein Trend, 
der durch sozio-ökonomischen Wandel und technologischen Fortschritt vorangetrieben wird, mit der Folge einer intensiven funktionalen Verflechtungen zwischen Kernstadt und Umland.

Von Continental Automotive GmbH kommt der Bericht "Vernetzung und Systemarchitektur - in weiser Voraussicht: Vernetzung und Datenfusion machen das Fahren sicherer, effizienter und komfortabler". Der darin beschriebene elektronische Horizont ist ein im Fahrzeug durch Verarbeitung zusätzlicher Streckeninformationen erstelltes dreidimensionales Profil der Fahrtstrecke. Das ermöglicht dem Fahrer frühe situativ optimierte Entscheidungen und Bordsystemen intelligente Fahrstrategien.

AlT Austrian Institute of Technology GmbH stellt "Gütermobilität 4.0: Vernetzt und elektrisch - Logistiktrends gestalten und nutzen" vor. Mit ihren richtungsweisenden Ansätzen kann zukünftig der derzeit ca. $20 \%$ des städtischen Verkehrs ausmachende, stetig ansteigende Gütertransport durch das Zusammenwirken von innovativen Elektrofahrzeugen mit entsprechenden Logistikkonzepten effizienter gestaltet werden.

Von NXP Semiconductors Austria GmbH kommt der Bericht "NXP to Showcase Connected Car of the Future", in welchem die Technologieführerschaft von NXP für lückenlose und sichere Verbindungen bei autonomem Fahren anhand des Einsatzes bei zukünftigen Mobilkonzepten der Concept Cars der Schweizer Rinspeed AG dargestellt wird. Dabei wird aufgezeigt, wie Fahrer autonomes Fahren erleben und in der Welt von morgen smarten und sicheren Zugriff auf ihr Auto haben.
Die ENIO GmbH berichtet in ihrem Beitrag „E-Mobilität demnächst ein Massenthema! Barrierefreier Zugang zum Elektroladen mit Kreditkarte und App und die Einbettung in die Gesamtenergiewirtschaft sind die heutigen Themen", wie sich ein notwendiger Umstieg auf E-Mobilität durch Benutzerfreundlichkeit, verbunden mit einer energiewirtschaftlichen Gesamtsicht, erfolgreich gestalten lässt.

Über unsere zukünftigen Lebensräume, etwa in vernetzten, digitalen Städten zur Abdeckung unserer Mobilitätserfordernisse, wurde und wird also viel nachgedacht. Besonderes Augenmerk ist bei der Einführung innovativer Lösungen auf das Zusammenwirken mit bestehenden Systemen zu legen. Erfreulicherweise wird der Übergang auf zukünftige Szenarien oftmals in die Betrachtungen mit einbezogen, und von vielen unbemerkt ist manches bereits im Fluss. Jedenfalls lässt die große inhaltliche Breite sowie die absehbare Komplexität des Übergangs auf Mobility 4.0 und die Digitalisierung des Verkehrs viele weitere interessante Forschungs- und Entwicklungsergebnisse erwarten.

Für diesen Themenschwerpunkt der e\&i wünschen wir Ihnen eine spannende Lektüre.

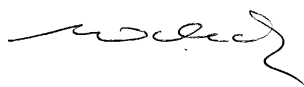

Helmut Malleck

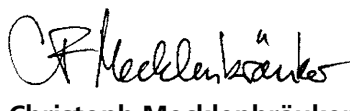

Christoph Mecklenbräuker 